\title{
On the group of zero-cycles of holomorphic symplectic varieties
}

\begin{abstract}
Alina Marian and Xiaolei Zhao
Abstract. For a moduli space of Bridgeland-stable objects on a K3 surface, we show that the Chow class of a point is determined by the Chern class of the corresponding object on the surface. This establishes a conjecture of Junliang Shen, Qizheng Yin, and the second author.

Keywords. K3 surface; moduli spaces of sheaves; Chow groups

2010 Mathematics Subject Classification. 14C25; 14D20; 14J28

[Français]

Sur le groupe des zéro-cycles des variétés symplectiques holomorphes

Résumé. Pour un espace de modules d'objets stables au sens de Bridgeland sur une surface K3, nous montrons que la classe de Chow d'un point est déterminée par la classe de Chern de l'objet correspondant sur la surface. Ceci établit une conjecture de Junliang Shen, Qizheng Yi et du second auteur.
\end{abstract}

Received by the Editors on May 23, 2019, and in final form on January 8, 2020.

Accepted on February 6, 2020.

Alina Marian

Department of Mathematics, Northeastern University

e-mail: a.marian@neu.edu

Xiaolei Zhao

Department of Mathematics, University of California, Santa Barbara

e-mail: xlzhao@ucsb.edu

(C) by the author(s)

This work is licensed under http://creativecommons.org/licenses/by-sa/4.0/ 
A Chow-theoretic study of holomorphic symplectic varieties of K3 type was undertaken in [SYZ]. The article considered moduli spaces of Bridgeland-stable sheaf complexes on a smooth projective K3 surface, and proposed to understand rational equivalence of zero-cycles on the moduli space in terms of the cycle structure of the underlying surface. We prove the following result conjectured in [SYZ].

Theorem. Let $X$ be a smooth projective $K 3$ surface. For a primitive $v \in H^{\star}(X, \mathbb{Z})$, and a $v$-generic stability condition $\sigma$, let $\mathrm{M}_{\sigma}(v)$ be the moduli space of $\sigma$-stable complexes on $X$ of Mukai vector $v$. Let $F_{1}$ and $F_{2}$ be two points in $\mathrm{M}_{\sigma}(v)$. Then

$$
\left[F_{1}\right]=\left[F_{2}\right] \in C H_{0}\left(\mathrm{M}_{\sigma}(v)\right) \Longleftrightarrow c_{2}\left(F_{1}\right)=c_{2}\left(F_{2}\right) \in C H_{0}(X) .
$$

As noted in [SYZ], if $F_{1}$ and $F_{2}$ have the same Chow class on $\mathrm{M}_{\sigma}(v)$, then by restricting a quasi-universal family on $M_{\sigma}(v) \times X$ to the two points one concludes that the Chern characters, hence the second Chern classes of $F_{1}$ and $F_{2}$ are equal in the Chow ring of $X$.

We show now that the existence of a quasi-universal family also establishes the other direction. Assume in fact for simplicity that $\mathrm{M}_{\sigma}(v)$ admits a universal family

$$
\mathcal{E} \rightarrow \mathrm{M}_{\sigma}(v) \times X .
$$

This restriction is certainly not essential, as we will discuss shortly. Denote the two projections by

$$
\pi: \mathrm{M}_{\sigma}(v) \times X \rightarrow \mathrm{M}_{\sigma}(v) \text { and } \rho: \mathrm{M}_{\sigma}(v) \times X \rightarrow X .
$$

The main role in the argument is played by the $\pi$-relative extension complex

$$
\mathrm{W}_{F}=\mathcal{E} x t_{\pi}^{\bullet}\left(\mathcal{E}, \rho^{\star} F\right)[1] \text { on } \mathrm{M}_{\sigma}(v),
$$

which is well-suited to detect in its Chern classes the Chow class of the point $F \in \mathrm{M}_{\sigma}(v)$. For any $E \in \mathrm{M}_{\sigma}(v)$ not isomorphic to $F$, by stability and duality we have

$$
\operatorname{Ext}^{0}(E, F)=0 \text { and } \operatorname{Ext}^{2}(E, F)=0 \text { on } X .
$$

In this situation, the middle group has constant dimension

$$
\operatorname{dim} \operatorname{Ext}^{1}(E, F)=\langle v, v\rangle,
$$

where the last quantity is the Mukai self-pairing of $v$. Note that the dimension of the moduli space is

$$
m=\langle v, v\rangle+2=\operatorname{dim} \mathrm{M}_{\sigma}(v) \text {. }
$$

On $M_{\sigma}(v) \backslash\{F\}$, we see therefore that $W_{F}$ is a locally free sheaf of rank $m-2$. This implies that on $M_{\sigma}(v)$ we have:

$$
\begin{aligned}
c_{m-1}\left(\mathrm{~W}_{F}\right) & =0, \\
c_{m}\left(\mathrm{~W}_{F}\right) & =\text { const } \cdot[F] .
\end{aligned}
$$

The constant can be determined by a Thom-Porteous calculation using a locally free complex isomorphic to $\mathrm{W}_{F}$. We simply have, in fact:

Proposition. The top Chern class of the complex $\mathrm{W}_{F}$ is

$$
c_{m}\left(\mathrm{~W}_{F}\right)=[F] .
$$

The proposition gives the theorem via Grothendieck-Riemann-Roch. As

$$
\operatorname{chW}_{F}=-\pi_{\star}\left(\operatorname{ch} \mathcal{E}^{\vee} \cdot \rho^{\star} \operatorname{ch} F \cdot \rho^{\star} \operatorname{td} X\right),
$$

we have in Chow,

$$
\operatorname{ch} F_{1}=\operatorname{ch} F_{2} \text { on } X \Longrightarrow \operatorname{chW}_{F_{1}}=\operatorname{chW}_{F_{2}} \text { on } \mathrm{M}_{\sigma}(v) \Longrightarrow c_{m}\left(\mathrm{~W}_{F_{1}}\right)=c_{m}\left(\mathrm{~W}_{F_{2}}\right) \text { on } \mathrm{M}_{\sigma}(v) \text {. }
$$


The Chern characters on $\mathrm{M}_{\sigma}(v)$ require rational coefficients. As the moduli space has trivial first cohomology group, Roitman's theorem $[\mathrm{Bl}],[\mathrm{R}]$ ensures that $\mathrm{CH}_{0}\left(\mathrm{M}_{\sigma}(v)\right)$ is torsion-free, hence the equality of the top Chern classes holds over $\mathbb{Z}$.

The proposition is not a new result, since the extension complex $W_{F}$ repeatedly played a role in the development of moduli theory of sheaves on K3 surfaces. Allowing $F$ to vary in moduli, one obtains the complex

$$
\mathrm{W}=\mathcal{E} x t_{\pi}^{\bullet}(\mathcal{E}, \mathcal{F})[1] \text { on } \mathrm{M}_{\sigma}(v) \times \mathrm{M}_{\sigma}(v),
$$

where $\pi: \mathrm{M}_{\sigma}(v) \times \mathrm{M}_{\sigma}(v) \times X \rightarrow \mathrm{M}_{\sigma}(v) \times \mathrm{M}_{\sigma}(v)$ is the projection, and $\mathcal{E}, \mathcal{F}$ are the universal objects pulled back to the triple product using the first and second factors respectively. This setting was considered in $[\mathrm{Ma}]$ when $\mathrm{M}_{\sigma}(v)$ parametrizes stable sheaves. In this situation, the main theorem in [Ma] asserts

$$
c_{m}(\mathrm{~W})=\Delta \in C H_{m}\left(\mathrm{M}_{\sigma}(v) \times \mathrm{M}_{\sigma}(v)\right) .
$$

This diagonal formula holds unsurprisingly for any stability condition $\sigma$, as we now indicate. The first step in [Ma] toward establishing the formula in the sheaf case is to write an explicit geometric threeterm complex of locally free sheaves which is isomorphic to the extension complex W. It is clear that for any generic stability condition $\sigma, \mathrm{W}$ is isomorphic to a three-term complex of locally free sheaves in $\mathrm{D}^{b}\left(\mathrm{M}_{\sigma}(v) \times \mathrm{M}_{\sigma}(v)\right)$

$$
A_{0} \stackrel{\alpha}{\rightarrow} A_{1} \stackrel{\beta}{\rightarrow} A_{2},
$$

where $A_{1}$ is placed in homological degree 0. This is true on general grounds [BM, Proposition 5.4] as long as for all points $x=(E, F) \in \mathrm{M}_{\sigma}(v) \times \mathrm{M}_{\sigma}(v)$, the vanishings

$$
H_{i}\left(\mathrm{~W} \stackrel{L}{\otimes} \mathcal{O}_{x}\right)=0 \text { for } i \neq-1,0,1
$$

hold. As we have $\mathrm{W}^{L} \otimes \mathcal{O}_{x}=\operatorname{Ext}_{X}^{\bullet}(E, F)[1]$, and $E, F$ both belong to the heart of the stability condition $\sigma$, this is true.

Note next that for the highest extension sheaf $\mathcal{E} x t_{\pi}^{2}(\mathcal{E}, \mathcal{F})$, cohomology and base change commute, so this sheaf is a line bundle supported on the diagonal $\Delta$. By contrast, $\mathcal{E} x t_{\pi}^{0}(\mathcal{E}, \mathcal{F})$ is torsion-free hence zero. For the above locally free complex, the map $\alpha$ is thus injective, while the cokernel of $\beta$ is a line bundle supported on the diagonal. Furthermore, Grothendieck-Verdier duality gives

$$
\mathrm{W}^{\vee}=\mathcal{E} x t_{\pi}^{\bullet}(\mathcal{F}, \mathcal{E})[1]
$$

so the cokernel sheaf of $\alpha^{\vee}: A_{1}^{\vee} \rightarrow A_{0}^{\vee}$ is also supported as a line bundle on $\Delta$.

The following general lemma proven in [Ma] by a suitable Thom-Porteous calculation then yields the diagonal formula (1) for any generic stability condition $\sigma$.

Lemma ([Ma], Lemma 4). Let

$$
A_{0} \stackrel{\alpha}{\rightarrow} A_{1} \stackrel{\beta}{\rightarrow} A_{2}
$$

be a complex of locally free sheaves on a smooth projective variety $M$, such that

(1) The cohomology sheaves satisfy $\mathcal{H}_{0}=0$ and $\mathcal{H}_{2}$ is a line bundle supported on a smooth subvariety $Z$ of even codimension $m$.

(2) The cokernel sheaf of $\alpha^{\vee}: A_{1}^{\vee} \rightarrow A_{0}^{\vee}$ is also supported as a line bundle on $Z$.

(3) The ranks satisfy $a_{1}-a_{0}-a_{2}=m-2 \geq 0$.

Then $c_{m}\left(A_{1}-A_{0}-A_{2}\right)=[Z]$. 
Since for any $F \in \mathrm{M}_{\sigma}(v)$, the complex $\mathrm{W}_{F}$ is the (derived) pullback

$$
\mathrm{W}_{F}=\iota_{F}^{\star} \mathrm{W}
$$

under the inclusion

$$
\iota_{F}: \mathrm{M}_{\sigma}(v) \simeq \mathrm{M}_{\sigma}(v) \times F \hookrightarrow \mathrm{M}_{\sigma}(v) \times \mathrm{M}_{\sigma}(v),
$$

the proposition is simply obtained from the diagonal formula (1) by pullback of $c_{m}(\mathrm{~W})$.

The moduli space $M_{\sigma}(v)$ does not generally admit a universal family. Nevertheless, as explained in [Mu, Theorem A.5], and later [Ma, Section 3], a universal object can be glued together over $\mathbb{P} \times X$, where $\mathbb{P} \rightarrow \mathrm{M}_{\sigma}(v)$ is a suitable projective bundle of a certain rank $N$. As further shown in [KLS, Lemma 4.2], for any point $F \in \mathrm{M}_{\sigma}(v)$, by taking the intersection of $\mathbb{P}$ with $N$ generic hyperplanes, one obtains a smooth projective variety $\widetilde{M}$ parametrizing a family

$$
\mathcal{E} \rightarrow \widetilde{M} \times X
$$

of $\sigma$-stable objects in $\mathrm{D}^{b}(X)$, such that the classifying morphism $f: \widetilde{M} \rightarrow \mathrm{M}_{\sigma}(v)$ is surjective, generically finite, and étale over a neighborhood of $F$.

Considering the $\pi$-relative extension complex

$$
\mathrm{W}_{F}=\mathcal{E} x t_{\pi}^{\bullet}\left(\mathcal{E}, \rho^{\star} F\right)[1] \text { on } \widetilde{M},
$$

the argument above gives

$$
f_{*} c_{m}\left(\mathrm{~W}_{F}\right)=n[F],
$$

where $n$ is the generic degree of $f$. Since $\mathrm{CH}_{0}\left(\mathrm{M}_{\sigma}(v)\right)$ is torsion-free, the theorem follows from this formula in conjunction with Grothendieck-Riemann-Roch.

Remark 1. In the classic paper $[\mathrm{Mu}]$, for isotropic Mukai vectors $v$, the author used $\mathrm{W}_{F}$ to establish irreducibility of the two-dimensional moduli space $M_{\sigma}(v)$. An argument with the same flavor was later made in [KLS] to show irreducibility in a more general setting. In a different direction, the aim in [Ma] is to represent the diagonal in terms of Chern classes of the universal sheaves, which entails that the cohomology of the moduli space is generated by the Künneth components of the universal Chern classes. The origin of this idea can in turn be traced back to the earlier work [Bel], [ES].

The subring of $\mathrm{CH}_{\star}\left(\mathrm{M}_{\sigma}(v)\right)$ generated in a suitable sense by the universal Chern classes is important for understanding the cycle class map to cohomology along the lines of [Be2], [V1].

Remark 2. The argument above gives the same statement for abelian surfaces. For surfaces with effective anti-canonical divisors (when $\mathrm{Ext}^{2}$ vanishes by Serre duality), the proposition reads

$$
c_{m}\left(\mathrm{~W}_{F}\right)=(-1)^{m}[F]
$$

and the proof is completely similar.

Remark 3. The consequences of the theorem were investigated in [SYZ]. In particular, the result implies the existence of algebraic coisotropic subvarieties $q_{i}: Z_{i} \rightarrow B_{i}$ for every $i$, where $Z_{i}$ is a subvariety of codimension $i$ in $\mathrm{M}_{\sigma}(v)$, and the generic fibers of $q_{i}$ are constant cycle subvarieties of dimension $i$. The existence of such varieties was conjectured in [V2] for any holomorphic symplectic variety $M$, and was used to construct a Beauville-Voisin filtration on $\mathrm{CH}_{0}(M)$. Another filtration on $\mathrm{CH}_{0}\left(\mathrm{M}_{\sigma}(v)\right)$ was constructed in [SYZ], and was shown to be related to the one in [V2] assuming the theorem. 


\section{Acknowledgements}

We thank Dragos Oprea for useful related discussions. A. M. was supported by the NSF through grant DMS 1601605. She thanks Davesh Maulik and the MIT mathematics department for their hospitality during the fall semester 2017. X. Z. is partially supported by the Simons Collaborative Grant 636187.

\section{References}

[Bel] A. Beauville, Sur la cohomologie de certains espaces de modules de fibrés vectoriels. In: Geometry and analysis (Bombay, 1992), pp. 37-40, Tata Inst. Fund. Res., Bombay, 1995.

[Be2] A. Beauville, On the splitting of the Bloch-Beilinson filtration. In: Algebraic cycles and motives. Vol. 2, pp. 38-53, London Math. Soc. Lecture Note Ser., vol. 344, Cambridge Univ. Press, Cambridge, 2007.

[Bl] S. Bloch, Torsion algebraic cycles and a theorem of Roitman, Compositio Math. 39 (1979), no. 1, 107-127.

[BM] T. Bridgeland and A. Maciocia, Fourier-Mukai transforms for K3 and elliptic fibrations, J. Algebraic Geom. 11 (2002), no. 4, 629-657.

[ES] G. Ellingsrud and S. A. Strømme, Towards the Chow ring of the Hilbert scheme of $\mathbf{P}^{2}, \mathrm{~J}$. Reine Angew. Math. 441 (1993), 33-44.

[KLS] D. Kaledin, M. Lehn, and Ch. Sorger, Singular symplectic moduli spaces, Invent. Math. 164 (2006), no. 3, 591-614.

[Ma] E. Markman, Generators of the cohomology ring of moduli spaces of sheaves on symplectic surfaces, J. Reine Angew. Math. 544 (2002), 61-82.

[Mu] S. Mukai, On the moduli space of bundles on K3 surfaces. I. In: Vector bundles on algebraic varieties (Bombay, 1984), pp. 341-413, Tata Inst. Fund. Res. Stud. Math., vol. 11, Tata Inst. Fund. Res., Bombay, 1987.

[R] A. A. Roitman, The torsion of the group of O-cycles modulo rational equivalence, Ann. of Math. (2) 111 (1980), no. 3, 553-569.

[SYZ] J. Shen, Q. Yin, and X. Zhao, Derived categories of K3 surfaces, O'Grady's filtration, and zero-cycles of holomorphic symplectic varieties, Compositio Math. 156 (2020), no. 1, 179-197.

[V1] C. Voisin, On the Chow ring of certain algebraic hyper-Kähler manifolds, Pure Appl. Math. Q. 4 (2008), no. 3, Special Issue: In honor of Fedor Bogomolov. Part 2, 613-649.

[V2] C. Voisin, Remarks and questions on coisotropic subvarieties and O-cycles of hyper-Kähler varieties. In: K3 surfaces and their moduli, pp. 365-399, Progr. Math., vol. 315, Birkhäuser/Springer, Cham, 2016. 